The patients in this study had a high vaginal delivery rate with low neonatal morbidity despite very low platelet counts in some mothers and babies.

\section{PM.35 CHANGING FROM 24-HOUR URINARY PROTEIN COLLECTIONS TO PROTEIN-CREATININE RATIOS: AN AUDIT OF ONE HOSPITAL'S EXPERIENCE OF TRYING TO REDUCE ADMISSION RATES FOR PRE-ECLAMPSIA}

doi:10.1136/archdischild-2013-303966.117

JL Sinclair, W Ogunnoiki, G Lawson. Tunbridge Wells Hospital, Maidstone and Tunbridge Wells NHS Trust, Tunbridge Wells, UK

Background Traditionally, 24-hour urinary protein collection has been used to quantify proteinuria in pre-eclampsia. There has been a move in recent years towards a "spot test" protein creatinine ratio (PCR) instead. The study hospital changed to PCR in December 2011. The audit was carried out to cheque that the department was following local and NICE Hypertension in Pregnancy guidelines and to see if there was a reduction in antenatal admission rates.

Methods The retrospective audit was carried out over two months. Data was collected from two periods - October to November 2011 (before introduction of PCR) and January to March 2012 (after introduction of PCR). Cases were identified from biochemistry department records. Data was collected on appropriate ordering and processing of tests, admission rates and length of stay in hospital. The ordering and processing of tests were compared with $95 \%$ standards. Admission rates were compared using Fisher's exact test and an odds ratio calculated. Lengths of stay were compared using a one-tailed Mann Whitney U test.

Results Patients from the PCR group were significantly less likely to be admitted to hospital ( $p=0.017$, odds ratio 0.2 ) and if admitted, length of stay was significantly shorter $(p=0.027)$.

Conclusions The significantly reduced admission rates and length of stay associated with the PCR group suggests that the diagnostic move from 24-hour urinary protein collection to PCR was beneficial to the department.

\section{PM.36 RESOURCE IMPLICATIONS OF CONVERTING FROM A WHO/ADA HYBRID TO IADPSG CRITERIA FOR DIAGNOSING GDM IN A UK UNIVERSITY HOSPITAL}

doi:10.1136/archdischild-2013-303966.118

R Anthony, P Angala, A lkomi, R Khan, S Kiss. Basildon and Thurrock University Hospital, Basildon, Essex, UK

Background In the UK, two studies have reported discordant findings in predicting the impact of International Association of the Diabetes and Pregnancy Study Group (IADPSG) criteria on the number of GDM cases ( $3 \%$ vs. $114 \%$ increase). This disparity warrants further evaluation. Our unit offers a 3 point GTT utilising the WHO thresholds for fasting and 2 hrs, American Diabetes Association (ADA) threshold for $1 \mathrm{hr}$ and like the IADPSG requires only one criterion for a positive diagnosis. Our unique access to this type of OGTT data makes us well placed to forecast the minimum impact on services.

Aims To ascertain the number of women diagnosed as GDM using WHO, WHO/ADA and IADPSG criteria. To determine the resource implications of IADPSG criteria.

Methods A retrospective study of 2905 OGTT results of women delivered in our unit between $1^{\text {st }}$ January 2009 and $31^{\text {st }}$ December 2011.
Results The numbers of women diagnosed with GDM were 327, 454 and 528 using WHO, WHO/ADA and IADPSG criteria respectively. This shows IADPSG criteria would lead to a $16.3 \%$ increase in our number of GDM cases equating to 25 extra cases/year. Had we been reliant on just WHO criteria, adopting IADPSG criteria would lead to a $61.4 \%$ increase, equating to 67 extra cases/year.

Conclusions UK units offering a 2 point WHO GTT should expect a $>60 \%$ increase in GDM numbers with IADPSG implementation. On the contrary, units already offering a 3 point WHO/ADA hybrid should anticipate a less drastic $16 \%$ increase.

\section{PM.37 EVALUATING THE SAFETY OF IVC PHILTRE USE DURING PREGNANCY: A CASE SERIES}

doi:10.1136/archdischild-2013-303966.119

${ }^{1} \mathrm{LK}$ Warrander, ${ }^{2,3} \mathrm{AE}$ Heazell, ${ }^{4} \mathrm{~N}$ Chalmers, ${ }^{3} \mathrm{~L}$ Byrd. ${ }^{1}$ Foundation Year 2 trainee, Central Manchester Univeristy Hospitals NHS Foundation Trust, Manchester, UK; ${ }^{2}$ Maternal and Fetal Health Research Centre, University of Manchester, Manchester, UK; ${ }^{3}$ Department of Obstetrics, St Mary's Hospital, Central Manchester University Hospitals NHS Foundation Trust, Manchester, UK; ${ }^{4}$ Department of Radiology, Manchester Royal Infirmary, Central Manchester University Hospitals NHS Foundation Trust, Manchester, UK

Pregnancy has been found to be associated with a 4-5 fold increase in the risk of venous thromboembolism (VTE), due to various physiological changes[1] and VTE has been the leading cause of direct maternal mortality in the UK over the past decade[2]. Management of VTE using an inferior vena cava (IVC) philtre is recommended by the Royal College of Obstetricians and Gynaecologists in women in the perinatal period with iliac vein VTE to reduce the risk of PE, or with proven DVT and continuing PE despite adequate anticoagulation. However, little data currently exists on the safety of IVC philtres during pregnancy.

Data were collected from women who underwent IVC philtre insertion for a VTE during pregnancy (January 2000 - September 2012) in the interventional radiology department at Central Manchester University Hospitals, Manchester.

Nine patients were initially identified, of which six had complete delivery data available. $67 \%(n=6)$ of patients had an acute VTE late in the third trimester, requiring philtre insertion and $56 \%$ $(n=5)$ also had a previous history of VTE. All patients delivered at term; one case was delivered by emergency caesarean section, and the remainder achieved vaginal deliveries. All infants were appropriately grown at birth and $83 \%(n=5)$ had 5 minute APGAR scores of 10 . There was one case of philtre retrieval failure.

This case series is concordant with other similar series, and suggests no detrimental effects on pregnancy. However, more research is needed to evaluate the long-term safety profile of IVC philtre use in pregnancy.

\section{REFERENCES}

1. Bourjeily et al. Pulmonary embolism in pregnancy. Lancet 2010;375(9713): 500-12.

2. CEMACH, Confidental Enquiry into Maternal and Child Health. Perinatal Mortality 2005: England, Wales and Northern Ireland. 2007: London.

\section{PM.38 CONDITIONS TRIGGERING LOCAL INCIDENT REVIEWS IN UK HOSPITAL MATERNITY UNITS}

doi:10.1136/archdischild-2013-303966.120

A Shah, C McClymont, M Knight. National Perinatal Epidemiology Unit, University of Oxford, Oxford, UK

Background In countries such as the UK where maternal deaths are rare, reviews of other severe complications can provide an additional perspective to help learn lessons to improve future care. The aim of this study was to identify the types of incidents which 